\title{
The Mathematical Foundations of Quantum Thermodynamics
}

\author{
Akira Suzuki' ${ }^{1}$ Hisao Taira² \\ ${ }^{1}$ Faculty of Science, Tokyo University of Science, Shinjyuku-Ku, Tokyo, Japan \\ ${ }^{2}$ Faculty of Education, Hokkaido University of Education, Kita-Ku, Sapporo, Japan \\ Email: asuzuki@rs.tus.ac.jp, taira.hisao@s.hokkyodai.ac.jp
}

How to cite this paper: Suzuki, A. and Taira, H. (2020) The Mathematical Foundations of Quantum Thermodynamics. Journal of Modern Physics, 11, 517-527. https://doi.org/10.4236/jmp.2020.114034

Received: February 10, 2020

Accepted: April 5, 2020

Published: April 8, 2020

Copyright (c) 2020 by author(s) and Scientific Research Publishing Inc. This work is licensed under the Creative Commons Attribution International License (CC BY 4.0).

http://creativecommons.org/licenses/by/4.0/

\begin{abstract}
It is proposed a representation of the basic laws (i.e. the zeroth, first, second and third laws) in thermodynamics for quantum systems in the pure and mixed ensembles, respectively. We show that the basic laws are represented by parameters that specify respective quantum states. The parameters are the elements of the thermodynamic state space $\mathcal{M}_{\theta}$ and the state space $\mathcal{M}_{\vartheta}$ of the mixed ensemble for quantum systems. The introduction of such parameters is based on a probabilistic nature of quantum theory. Consistency between quantum theory and classical thermodynamics is preserved throughout the formulation for the representation of the thermodynamical laws in quantum systems (quantum thermodynamics). The present theory gives the mathematical foundations of quantum thermodynamics.
\end{abstract}

\section{Keywords}

Basic Laws in Thermodynamics, Thermodynamic State Space, Pure and Mixed States, Classical Thermodynamics, Quantum Thermodynamics, Transitive Law, Mixed Ensemble

\section{Introduction}

Thermodynmics is a universal theory not only for classical but for quantum systems. Classical thermodynamics has been well established by different approaches [1] [2] [3]. Above all, the theoretical importance of thermodynamical consideration in quantum systems (quantum thermodynamics) is emphasized in textbooks [4] [5]. When we consider the thermodynamics for quantum systems, the most important is the change in entropy since entropy is a constant of motion under the unitary transformation generated by a system Hamiltonian [6] [7]. The internal energy of the system plays the same role as temperature [8]. It 
should, however, be noticed that in quantum system, thermodynamic equibria cannot be described in terms of a parameter like a temperature as in classical system.

In classical thermodynamics, states of the system are represented by points on a thermodynamic state space. In quantum system, quantum states are expressed by the elements of a complex Hilbert space $\mathscr{K}$. Therefore, in quantum thermodynamics, the Hilbert space itself does not play the same role as the state space in classical thermodynamics. In quantum system, thermodynamic equibria cannot be described in terms of a parameter like a temperature as in classical system. To be more precise, respective thermodynamic states are represented by points on a thermodynamic state space $\mathcal{M}_{\theta}$, and a different point means other thermodynamic states.

In order to gain some insights into the relation between thermodynamic states and quantum states, we have to investigate the structure of thermodynamics (quantum thermodynamics) on the basis of mathematical foundations. Lieb and Yngvason made the mathematical structure of thermodynamics transparent by axiomatic approach [3]. Thermodynamics treats changes of thermodynamical quantities when a system changes from one thermodynamic state to another state. In quantum mechanics, quantum states are expressed by the elements of a complex Hilbert space $\mathscr{K}$. It should be however noted that the Hilbert space itself does not play the same role as the thermodynamic state space in classical thermodynamics. In other words, the thermodynamic state space is not the same state space. The present authors [9] started with introducing a set $\mathcal{M}_{\Psi}$ of the state vectors in $\mathscr{H}$ in order to obtain a suitable set which plays the same role as the thermodynamic state space in classical thermodynamics. That is, a set $\mathcal{M}_{\theta}$, which plays the same role as the thermodynamic state space, is introduced and the correspondence between $\mathcal{M}_{\Psi}$ and $\mathcal{M}_{\theta}$ must be derived. In short, we will see this treatment provides an insight into a correspondence between $\mathcal{M}_{\Psi}$ and $\mathcal{M}_{\theta}$.

In our previous paper [9], we showed that a representation of the basic laws in thermodynamics for quantum systems in pure states is constructed in terms of the parameters $\theta$ 's by which respective thermodynamic states corresponding to the respective quantum states of the system are represented. In this paper, we extend the previous theory for the representation of the thermodynamic quantum states applicable to the case for the mixed ensembles in quantum systems. We introduced parameters $\theta$ 's in $\mathcal{M}_{\theta}$, which could describe the thermodynamic temperature. The parameters $\theta$ 's make sense only on existence of the map $\mathcal{M}_{\Psi} \mapsto \mathcal{M}_{\theta}$, i.e., $\mathcal{M}_{\theta}$ is an injection of $\mathcal{M}_{\Psi}$ valid for a system in pure states.

To extend the range of applicability of the theory is interesting from the viewpoint of fundamental physics. In the previous study [9] we discussed thermodynamics for a system in pure quantum states and constructed the representation of basic laws of quantum thermodynamics in terms of the parameters $\theta$ s 
in $\mathcal{M}_{\theta}$. In this paper, we extend the theory and construct the formulas representing the basic laws of thermodynamics for a system in a mixed quantum ensemble. The formulation is based on the mathematical foundation by employing axiomatic approach [3].

We will introduce a state space $\mathcal{M}_{\vartheta}$ in order to take into account a mixed state just like we introduced $\mathcal{M}_{\theta}$ for a pure state. The parameter $\vartheta$ 's characterize the state vectors of the mixed states, where fractional population (viz., a probability of occurrence) of the quantum states is naturally introduced for state vectors. It should be noted that the parameters $\vartheta$ 's are the elements of $\mathcal{M}_{\vartheta}$ for a system in mixed quantum states and the map $\mathcal{M}_{\Psi} \mapsto \mathcal{M}_{\vartheta}$ should hold. We will show that a representation of the basic laws (Zeroth, First, Second and Third laws) in thermodynamics for quantum systems in a mixed state is described by making use of those parameters $\vartheta$ 's in $\mathcal{M}_{\vartheta}$.

This paper is organized as follows. In the next section, we present quantum thermodynamics, namely thermodynamics for quantum systems. In subsection 2.1, the zeroth law of quantum thermodynamics is formulated for the case where the quantum systems are in mixed quantum ensembles. We introduce thermodynamic state space $\mathcal{M}_{\theta}, \mathcal{M}_{\vartheta}$ respectively for pure and mixed quantum ensembles in order to relate $\mathcal{M}_{\theta}, \mathcal{M}_{\vartheta}$ to the quantum state space $\mathcal{M}_{\Psi}$. We discuss the connection between the elements in $\mathcal{M}_{\Psi}$ and those in $\mathcal{M}_{\theta}$ and $\mathcal{M}_{\vartheta}$, and formulate the first law of quantum thermodynamics in subsection 2.2. In subsection 2.3, we introduce an entropy function for the system of mixed states as a map on thermodynamic state space $\mathcal{M}_{\theta}$ and $\mathcal{M}_{\vartheta}$ for pure and mixed ensembles. We shall see that the entropy function ensures it is defined for all states in terms of $\theta$ 's $\left(\in \mathcal{M}_{\theta}\right)$ and $\vartheta$ 's $\left(\in \mathcal{M}_{\vartheta}\right)$. We will show that thermodynamic temperatures can be defined as a function of $\theta$ 's and $\vartheta$ 's for the system of mixed ensembles. In subsection 2.4, the third law of thermodynamics for the quantum systems of mixed states is briefly discussed. The absolute zero temperature is the state that the system is in a single quantum state. Finally, summary and concluding remarks are given in section 3.

\section{Thermodynamics for Systems in Mixed Quantum States}

In the previous paper [9], we have considered the case where thermodynamic quantum systems are in pure ensembles. The state of quantum systems in a pure ensemble is described by $\left|\Psi_{\alpha}\right\rangle,\left|\Psi_{\beta}\right\rangle$, etc., where subscripts denote the label of respective states of quantum system. The formulation of the present theory can be extended to a case where thermodynamic quantum systems are in mixed ensemble by introducing another parameter in order to describe those mixed ensembles of the quantum system.

Mixed ensembles are defined by

$$
\rho:=\left(\left|\Psi_{\alpha}\right\rangle,\left|\Psi_{\beta}\right\rangle,\left|\Psi_{\gamma}\right\rangle, \cdots ; w_{\alpha}, w_{\beta}, w_{\gamma}, \cdots\right),
$$

where fractional populations $w_{j}\left(0<w_{j} \leq 1\right.$ and $\left.\sum w_{j}=1, j=\alpha, \beta, \gamma, \cdots\right)$ 
describes a probability of occurrence corresponding to a state vector $\left|\Psi_{j}\right\rangle$ [10]. This suggests that a mixed ensemble is characterized not only by a probability $w_{j}$ but also by a probability $p$ which is obtained from $|\Psi\rangle$ as shown in the proof of L1' (see Section 2.1). Therefore if we introduce a probability function $Q(\vartheta ; y)$ so as to satisfy $Q(\vartheta ; y=j)=\omega_{j}$, a mixed ensemble can be characterized by both $Q(\vartheta ; y)$ and $P(\theta ; x)$. Thus, a state of the thermodynamic quantum systems can be characterized by these two parameters: $\vartheta$ 's in $\mathcal{M}_{\vartheta}$ and $\theta$ 's in $\mathcal{M}_{\theta}$. It should be noticed that the origin of $\theta$ is a probabilistic nature of quantum mechanics but $\vartheta$ is due to the 'classical' probability of occurrence $\omega_{j}$ that the state vector is $\left|\Psi_{j}\right\rangle$. In this section, we derive a representation of zeroth, first, second, and third laws of thermodynamics for a quantum system in a mixed ensemble.

At first, we have to introduce the concept of an ensemble $w_{j}$ into the probability amplitude $a_{i}$ defined by $a_{i} \equiv\langle i \mid \Psi\rangle$ where $\langle i|=| i\rangle^{\dagger},(i=1,2,3, \cdots)$, is complete orthogonal basis. We define the probability amplitude in a mixed ensemble as follows:

$$
b_{i j} \equiv \sqrt{w_{j}}\left\langle i \mid \Psi_{j}\right\rangle
$$

The probability is thus given by

$$
p_{i j}=\left|b_{i j}\right|^{2}=w_{j}\left|\left\langle i \mid \Psi_{j}\right\rangle\right|^{2}=p_{i} w_{j} .
$$

Recall that $\left|\left\langle i \mid \Psi_{j}\right\rangle\right|^{2}$ is the usual quantum mechanical probability taken with respect to the respective state $i$. Equation (2) tells us that these probabilities must further be weighted by the corresponding fractional populations $w_{j}$. Notice how probabilistic concepts enter twice: first in $\left|\left\langle i \mid \Psi_{j}\right\rangle\right|^{2}$ for the quantum-mechanical probability for the respective state $i$ to be found in an eigenstate $\left|\Psi_{j}\right\rangle$, second in the probability factor $w_{j}$ for finding a quantum-mechanical state characterized by $\left\langle i \mid \Psi_{j}\right\rangle$ in the ensemble.

In the following subsections, we derive the representation of the basic laws of quantum thermodynamics for a system in mixed ensembles.

\subsection{The Zeroth Law of Quantum Thermodynamics}

The zeroth law of quantum thermodynamics for a system in mixed ensembles is expressed by making use of the parameter $\vartheta$ 's in $\mathcal{M}_{\vartheta}$. In order to represent the zeroth law, we need to introduce the Lemma L1' as follows:

L1': There exist parameters $\theta_{\alpha}, \theta_{\beta}, \cdots$ in $\mathcal{M}_{\theta}$ for pure quantum states and $\vartheta_{\alpha}, \vartheta_{\beta}, \cdots$ in $\mathcal{M}_{\vartheta}$ for mixed ensembles representing the respective state vectors, $\left|\Psi_{\alpha}\right\rangle,\left|\Psi_{\beta}\right\rangle, \cdots$ in $\mathcal{M}_{\Psi}$.

Proof of L1': We treat a case for a label $\alpha$. Other cases, $\beta, \gamma, \cdots$, could be proved in the same way. From Equation (2), one can obtain a sequence $\left\{\left|b_{1 j}^{\alpha}\right|^{2},\left|b_{2 j}^{\alpha}\right|^{2}, \cdots,\left|b_{i j}^{\alpha}\right|^{2}, \cdots\right\}$ with an ensemble $j$. By normalizing a state vector $\left|\Psi_{\alpha}\right\rangle$, the sequence satisfies the condition $\sum_{i=1}\left|b_{i j}^{\alpha}\right|^{2}=1$ and it is clear that 
$0 \leq\left|b_{i j}^{\alpha}\right|^{2} \leq 1$. Then each element of the sequence describes a definite probability for the respective state $i$ and a specific ensemble $j$. Hereafter, we shall omit superscript $\alpha$ for simplicity. Let us introduce a probability function $P(\theta ; x)$ so as to satisfy $P(\theta ; x=i)=\left|a_{i}\right|^{2}$ for any $i$. In $P(\theta ; x), \quad \theta$ is a parameter and $x$ is a random variable. We also introduce a probability function so as to satisfy $Q(\vartheta ; y=j)=w_{j}$ for any $j$. Therefore, the parameters $\theta_{k} \quad(k=\alpha, \beta, \gamma, \cdots)$ and $\vartheta_{k}(k=\alpha, \beta, \gamma, \cdots)$, both exist for the representation of a state vector $\left|\Psi_{k}\right\rangle$.

We note here that $\mathbf{L} 1^{\prime}$ ensures the existence of parameters $\theta_{k}$ 's and $\vartheta_{k}$ 's which correspond to respective state vectors $\left|\Psi_{k}\right\rangle$ 's in $\mathcal{M}_{\Psi}$ through probability functions $P\left(\theta_{k} ; x\right)$ 's and in $Q\left(\vartheta_{k} ; y\right)$ 's, respectively.

Now one can compare two quantum states in thermodynamic sense since those parameters $\theta$ 's and $\vartheta$ 's can be used to describe two or more systems being equivalent. This leads to the zeroth law of quantum thermodynamics. Let us prepare three systems, $\mathcal{M}_{\theta}^{\mathrm{A}}$ with $\mathcal{M}_{\vartheta}^{\mathrm{A}}, \mathcal{M}_{\theta}^{\mathrm{B}}$ with $\mathcal{M}_{\vartheta}^{\mathrm{B}}$ and $\mathcal{M}_{\theta}^{\mathrm{C}}$ with $\mathcal{M}_{\vartheta}^{\mathrm{C}}$, where superscripts indicate labels of respective systems. The zeroth law of quantum thermodynamics (equivalence relation among quantum states) is described by the following relation:

Let us first consider the following relation for the system in mixed ensembles:

$$
\text { If } \theta_{\alpha}^{\mathrm{A}}=\theta_{\beta}^{\mathrm{B}} \text { and } \vartheta_{\alpha}^{\mathrm{A}}=\vartheta_{\beta}^{\mathrm{B}} \text {, then }\left|\Psi_{\alpha}\right\rangle^{\mathrm{A}} \doteq\left|\Psi_{\beta}\right\rangle^{\mathrm{B}} \text {, }
$$

where $\theta_{\alpha}^{\mathrm{A}} \in \mathcal{M}_{\theta}^{\mathrm{A}}$ and $\theta_{\beta}^{\mathrm{B}} \in \mathcal{M}_{\theta}^{\mathrm{B}}, \vartheta_{\alpha}^{\mathrm{A}} \in \mathcal{M}_{\vartheta}^{\mathrm{A}}$ and $\vartheta_{\beta}^{\mathrm{B}} \in \mathcal{M}_{\vartheta}^{\mathrm{B}}$ respectively, and a symbol $\doteq$ here denotes that the state in the left-hand side is equivalent to the state in the right-hand side.

Proof of Equation (4): Let $\theta_{\alpha}^{\mathrm{A}}$ and $\theta_{\beta}^{\mathrm{B}}$ be the elements of $\mathcal{M}_{\theta}^{\mathrm{A}}$ and $\mathcal{M}_{\theta}^{\mathrm{B}}$, respectively. Furthermore, let $\vartheta_{\alpha}^{\mathrm{A}}$ and $\vartheta_{\beta}^{\mathrm{B}}$ be the elements of $\mathcal{M}_{\vartheta}^{\mathrm{A}}$ and $\mathcal{M}_{\vartheta}^{\mathrm{B}}$, respectively. By $\mathbf{L} 1^{\prime}$, it is clear that $\theta_{\alpha}^{\mathrm{A}}=\theta_{\beta}^{\mathrm{B}}$ and $\vartheta_{\alpha}^{\mathrm{A}}=\vartheta_{\beta}^{\mathrm{B}}$ imply $\left|\Psi_{\alpha}\right\rangle^{\mathrm{A}} \doteq\left|\Psi_{\beta}\right\rangle^{\mathrm{B}}$.

The zeroth law: We are now in a position to discuss some consequences obtained by introducing the parameters $\theta_{k}$ and $\vartheta_{k}$ to specify the corresponding thermodynamic states of the systems in mixed quantum ensembles. It is clear from Equation (4) that the transitive law holds:

If $\theta_{\alpha}^{\mathrm{A}}=\theta_{\beta}^{\mathrm{B}}$ with $\vartheta_{\alpha}^{\mathrm{A}}=\vartheta_{\beta}^{\mathrm{B}}$ and $\theta_{\beta}^{\mathrm{B}}=\theta_{\gamma}^{\mathrm{C}}$ with $\vartheta_{\beta}^{\mathrm{B}}=\vartheta_{\gamma}^{\mathrm{C}}$, then $\left|\Psi_{\alpha}\right\rangle^{\mathrm{A}} \doteq\left|\Psi_{\gamma}\right\rangle^{\mathrm{C}}$.

We have established a representation of the zeroth law of quantum thermodynamics (equivalence relation among quantum states). The zeroth law can be expressed in terms of parameters in $\mathcal{M}_{\theta}$ and $\mathcal{M}_{\vartheta}$. Equation (5) means that, in the mixed ensemble, the additional condition $\vartheta_{\alpha}^{\mathrm{A}}=\vartheta_{\beta}^{\mathrm{B}}$ is required for the equivalence relation among mixed ensembles.

\subsection{The First Law of Quantum Thermodynamics}

The first law of thermodynamics states that heat is a form of energy, and thermodynamic processes are therefore subject to the principle of conservation of energy, meaning that heat energy cannot be created or destroyed. In order to 
discuss the first law and obtain the representation of the first law of quantum thermodynamics, let us consider the internal energy of the system in mixed quantum ensembles.

The internal energy $U_{\mathrm{m}}$ of the system in mixed quantum states is given by the expectation value of Hamiltonian $\hat{\mathcal{H}}$ :

$$
U_{\mathrm{m}}=\langle\hat{\mathcal{H}}\rangle_{\mathrm{m}}=\sum_{i, j} p_{i j} E_{i}=\sum_{j} U_{j},
$$

where $p_{i j}=p_{i} w_{j}$ and $U_{j}=\sum_{i} p_{i j} E_{i}$ is the internal energy of the ensemble $j$. By calculating the total differential of Equation (6), we obtain

$$
d U_{\mathrm{m}}=\sum_{i, j}\left(E_{i} d p_{i j}+p_{i j} d E_{i}\right) \equiv \sum_{j}\left(d^{\prime} Q_{j}+d^{\prime} W_{j}\right) .
$$

Analogous to the case for a system in a pure ensemble [9], we identify $d^{\prime} Q_{j}=\sum_{i} E_{i} d p_{i j}$ and $d^{\prime} W_{j}=\sum_{i} p_{i j} d E_{i}$, respectively, for the heat transferred to the ensemble $j$ and for the work done on the ensemble $j$. The variation of the internal energy $U_{\mathrm{m}}$, that is $d U_{\mathrm{m}}$ for the mixed ensemble, is obtained by summing up all $d^{\prime} Q_{j}$ and $d^{\prime} W_{j}$ weighted by $w_{j}$ since $p_{i j}$ is equal to $p_{i} w_{j}$. It is clear that $d U_{\mathrm{m}}$ in Equation (7) is reduced to $d U=\sum_{i}\left(E_{i} d p_{i}+p_{i} d E_{i}\right)$, which is the case of a quantum system in a pure quantum ensemble since a pure quantum ensemble means $w_{j}=1$ only for particular $j$ and $w_{j^{\prime}}=0$ for $j^{\prime} \neq j$.

Distinguishing two kinds of transfer of energy, as heat and as thermodynamic work, adopted for thermodynamic processes, we prove that the equalities $d^{\prime} W_{j}=\sum_{i} p_{i j} d E_{i}$ and $d^{\prime} Q_{j}=\sum_{i} E_{i} d p_{i j}$ are true. Let us consider the small change in the outcome is given by

$$
d E_{i}=\frac{\partial E_{i}(L)}{\partial L} d L
$$

where $L$ is the work coordinate related to the work done on the system. Then

$$
\sum_{i} p_{i j} d E_{i}=\sum_{i} p_{i j} \frac{\partial E_{i}}{\partial L} d L
$$

where $p_{i j}=P(\theta ; x=i) Q(\vartheta ; y=j)$. Note that $p_{i j}$ is a function of the parameters $\theta$ and $\vartheta$. The probability $p_{i j}$ does not depend on $L$ but the energy $E_{i}$ of the quantum state $i$ does. Thus we can write Equation (9) as

$$
\sum_{i} p_{i j} d E_{i}=\sum_{i} \frac{\partial}{\partial L}\left(p_{i j} E_{i}\right) d L=\frac{\partial}{\partial L} U_{j} d L .
$$

A change in the internal energy of the system in a mixed ensemble is generally related to a force defined by

$$
F_{j} \equiv-\frac{\partial U_{j}(L)}{\partial L},
$$

so that Equation (10) and hence Equation (9) can be expressed as

$$
\sum_{i} p_{i j} d E_{i}=-F_{j} d L
$$

Thus, it is clear that the term $\sum_{i} p_{i j} d E_{i}$ corresponds to the work $d^{\prime} W_{j}$ done 
on the system described by ensemble $j$ and it is generally expressed by

$$
d^{\prime} W_{j}=\sum_{i} p_{i j} d E_{i}(L)=-F_{j} d L,
$$

where the definite probability $p_{i j}$ can then be replaced by the probability function $P(\theta ; x=i) Q(\vartheta ; y=j)$. Thereby, from Equations (7) and (13) we have $d U_{j}=d^{\prime} Q_{j}$ when $d^{\prime} W_{j}=0$. Then the term $\sum_{i} E_{i} d p_{i j}$ corresponds to the change in the internal energy of the system that occurs when no work is done; this is what we understand as heat flow.

It should be emphasized that the heat entering the system described by ensemble $j, d^{\prime} Q_{j}$, is expressed in terms of the variation of $p_{i j}=P(\theta ; x=i) Q(\vartheta ; y=j)$ while the work is done on the system described by ensemble $j, d^{\prime} W_{j}$, is expressed in terms of the variation of $E_{i}(L)$. Since $p_{i j}=P(\theta ; x=i) Q(\vartheta ; y=j)$, we can write $d p_{i j}$ as

$$
d p_{i j}=Q(\vartheta ; y=j) \frac{\partial P(\theta ; x=i)}{\partial \theta} d \theta+P(\theta ; x=i) \frac{\partial Q(\vartheta ; y=j)}{\partial \vartheta} d \vartheta .
$$

so that $d^{\prime} Q_{j}$ is expressed in terms of those parameters $\theta$ and $\vartheta$ :

$$
\begin{aligned}
& d^{\prime} Q_{j}=\sum_{i} E_{i} d p_{i j} \\
& =\sum_{i} E_{i}\left[Q(\vartheta ; y=j) \frac{\partial P(\theta ; x=i)}{\partial \theta} d \theta+P(\theta ; x=i) \frac{\partial Q(\vartheta ; y=j)}{\partial \vartheta} d \vartheta\right] .
\end{aligned}
$$

We would like to note that Equation (15) reduces to

$d^{\prime} Q=\sum_{i} E_{i} d p_{i}=\sum_{i} E_{i} \frac{\partial P(\theta ; x=i)}{\partial \theta} d \theta$ for a quantum system in a pure ensemble, where $Q(\vartheta ; y=j)=1$.

\subsection{The Second Law of Quantum Thermodynamics}

In this subsection, we will give a definition of entropy to describe the entropy principle (viz., the second law of thermodynamics) for quantum system described by a mixed state. The entropy principle states that adiabatic accessibility of any two states is described by an entropy inequality. Here we should refer to the adiabatic process since the second law treated here is defined for the process. The process is characterized by $d^{\prime} Q_{j}=0$ for mixed ensembles. This is ensured when $P(\theta ; x) Q(\vartheta ; y)$ remains unchanged throughout the process (see $\mathrm{Cl}^{\prime}$ and argument below). In other words, adiabatic process is a process such that $P(\theta ; x) Q(\vartheta ; y)$ remains unchanged. It should be noted that adiabatic process allows to change a value of $L$ since it affects only the work $d^{\prime} W_{j}$. This is consistent with adiabatic processes defined by Lieb and Yngvason [3].

Let us define an entropy function $S_{\mathrm{m}}$ for mixed ensembles as a map from the sets $\mathcal{M}_{\theta, L}$ and $\mathcal{M}_{\vartheta, L}$ to a real number $\mathcal{R}$ :

$$
S_{\mathrm{m}}: \mathcal{M}_{\theta, L}, \mathcal{M}_{\vartheta, L} \mapsto \mathcal{R} .
$$

We note that this general definition for entropy can describe all types of entropy functions including well known Boltzmann, Gibbs, and Shannon entro- 
pies. The entropy $S_{\mathrm{m}}$ defined by the map (16) is clearly a state quantity and ensures that $S_{\mathrm{m}}$ can be defined for all states (i.e. pure and mixed ensembles) in terms of $\theta$ and $\vartheta$.

In order to obtain a representation of the second law in terms of $\theta$ and $\vartheta$ for quantum system, it must be shown that determining parameters $\theta$ and $\vartheta$. In order to show this, let us show that $U_{\mathrm{m}}$ is specified by $\theta$ 's, $\vartheta$ 's and $L$ 's (i.e., the elements of $\mathcal{M}_{\theta, L}$ and $\mathcal{M}_{\vartheta, L}$ ).

First, we show that the following corollaries, $\mathrm{C1}^{\prime}$ and $\mathrm{C2}^{\prime}$, can be drawn:

C1': The internal energy $U_{\mathrm{m}}$ is specified by the respective parameters $\theta, \vartheta$ and $L$ :

$$
U_{\mathrm{m}}=U_{\mathrm{m}}(\theta, \vartheta, L) .
$$

Proof of $\mathrm{C1}^{\prime}$ : Once a maximal test is chosen for a fixed $L$, respective outcomes $E_{i} \quad(i=1,2, \cdots)$ for the system is uniquely determined and the definite probability $p_{i j}$ is then described as $P(\theta ; x=i) Q(\vartheta ; y=j)$ by the proof of $\mathbf{L} 1^{\prime}$. Therefore, it is clear that the internal energy is specified by the respective parameters $\theta, \vartheta$ and $L$.

We note that $\mathbf{C 1}$ ' states the internal energy $U_{\mathrm{m}}$ can be specified by those parameters, $\theta, \vartheta$ and $L$. We will omit $L$ in $U_{\mathrm{m}}(\theta, \vartheta, L)$ for simplicity when we consider a fixed $L$.

C2': $d^{\prime} Q_{j}=0$ implies the consequence of adiabatic equivalence for all ensembles w.r.t. each $j$.

Proof of C2': By the proof of L1', no change in the probability function implies that the absolute values of the expansion coefficients $\left|b_{i j}\right|$ 's remain the same. This implies $p_{i j}$ remains constant. Thus $d^{\prime} Q_{j}$ is equal to zero throughout the operation (process).

In the statement of $\mathbf{C 2}^{2}$, the consequence of adiabatic equivalence is as follows: if the system were isolated, the absolute values of the expansion coefficients $\left|b_{i j}\right|$ 's would remain constant. It should be noticed that the notion of heat arises only when the state (internal energy) of a system changes, where $d U_{j} \neq d^{\prime} W_{j}$. As in classical thermodynamics, heat in quantum system is also defined as a form of energy flow. Once the internal energy of a quantum system is well defined, heat is also well defined. Thus the following Lemma (L2') is established:

L2': There exist $U_{\mathrm{m}}$ 's specified by each element of $\mathcal{M}_{\theta, L}$ and $\mathcal{M}_{\vartheta, L}$.

Proof of L2': Without loss of generality, one can consider a fixed maximal test, where outcome of the maximal test is uniquely determined: The internal energy for mixed ensembles is thus represented in terms of those parameters $\theta$ 's and $\vartheta$ 's:

$$
U_{\mathrm{m}}=\sum_{i, j} p_{i j} E_{i}=\sum_{i, j} P\left(\theta_{\alpha} ; x=i\right) Q\left(\vartheta_{\alpha} ; y=j\right) E_{i},
$$

where $\theta_{\alpha}$ and $\vartheta_{\alpha}$ specify the internal energy $U_{\mathrm{m}}$. Therefore, $U_{\mathrm{m}}$ can be labeled as $U_{\mathrm{m} \alpha}$. As in the same way, respective internal energies, $U_{\mathrm{m} \beta}, U_{\mathrm{m} \gamma}, \cdots$, can be specified by $\theta_{\beta}, \theta_{\gamma}, \cdots$ and $\vartheta_{\beta}, \vartheta_{\gamma}, \cdots$.

Since the existence of correspondence between an internal energy $U_{\mathrm{m}}$ and 
parameters $\theta$ and $\vartheta$ was established by L2', we can obtain one-to-one correspondence between $S_{\mathrm{m}}(X)$ and $S_{\mathrm{m}}(\theta, \vartheta)$. This keeps consistency between an entropy function defined in the entropy principle (see Ref. [9], Section 2) and the statement (16) for a mixed ensemble of the system. We finally obtain a representation of the second law of quantum thermodynamics for a system in a mixed ensemble in terms of $\theta$ and $\vartheta$ :

$$
\theta_{\alpha} \prec \theta_{\alpha^{\prime}} \text { and } \vartheta_{\alpha} \prec \vartheta_{\alpha^{\prime}} \text { if and only if } S_{\mathrm{m}}\left(\theta_{\alpha}, \vartheta_{\alpha}\right) \leq S_{\mathrm{m}}\left(\theta_{\alpha^{\prime}}, \vartheta_{\alpha^{\prime}}\right) \text {. }
$$

This describes the entropy principle for quantum systems in mixed quantum ensembles.

For the case where a given arbitrary pair of states represented by $\left(\theta_{\alpha}, \theta_{\beta}\right)$ and $\left(\theta_{\alpha^{\prime}}, \theta_{\beta^{\prime}}\right)$, the following relation holds [9]:

$$
\left(\theta_{\alpha}, \theta_{\beta}\right) \prec\left(\theta_{\alpha^{\prime}}, \theta_{\beta^{\prime}}\right) \text { if and only if } S\left(\theta_{\alpha}\right)+S\left(\theta_{\beta}\right) \leq S\left(\theta_{\alpha^{\prime}}\right)+S\left(\theta_{\beta^{\prime}}\right) \text {, }
$$

where $\theta_{j}, j=\alpha, \beta, \cdots$, is the element of the state space $\mathcal{M}_{\theta} \times \mathcal{M}_{\theta}$.

From L2', we can immediately obtain the relation for any pairs of states represented by $\left(\theta_{\alpha}, \theta_{\beta}\right),\left(\vartheta_{\alpha}, \vartheta_{\beta}\right)$ and $\left(\theta_{\alpha^{\prime}}, \theta_{\beta^{\prime}}\right),\left(\vartheta_{\alpha^{\prime}}, \vartheta_{\beta^{\prime}}\right)$ :

$$
\begin{aligned}
& \left(\theta_{\alpha}, \theta_{\beta}\right) \prec\left(\theta_{\alpha^{\prime}}, \theta_{\beta^{\prime}}\right) \text { and }\left(\vartheta_{\alpha}, \vartheta_{\beta}\right) \prec\left(\vartheta_{\alpha^{\prime}}, \vartheta_{\beta^{\prime}}\right) \\
& \text { if and only if } S\left(\theta_{\alpha}, \vartheta_{\alpha}\right)+S\left(\theta_{\beta}, \vartheta_{\beta}\right) \leq S\left(\theta_{\alpha^{\prime}}, \vartheta_{\alpha^{\prime}}\right)+S\left(\theta_{\beta^{\prime}}, \vartheta_{\beta^{\prime}}\right) \text {, }
\end{aligned}
$$

where $\theta_{k}$ and $\vartheta_{k}, k=\alpha, \beta, \cdots$, are the elements of the state space $\mathcal{M}_{\theta} \times \mathcal{M}_{\theta}$ and $\mathcal{M}_{\vartheta} \times \mathcal{M}_{\vartheta}$, respectively. The statement (21) means that $\left(\theta_{\alpha^{\prime}} ; \vartheta_{\alpha^{\prime}}, \theta_{\beta^{\prime}} ; \vartheta_{\beta^{\prime}}\right)$ is adiabatically accessible from $\left(\theta_{\alpha} ; \vartheta_{\alpha}, \theta_{\beta} ; \vartheta_{\beta}\right)$.

Since we established $S_{\mathrm{m}}$ for the quantum system as a function of parameters $\theta$ and $\vartheta$, one can define thermodynamic temperature $T$ as a function of $\theta$ and $\vartheta: T=T(\theta, \vartheta)$.

\subsection{The Third Law of Quantum Thermodynamics}

Let us briefly discuss the third law of thermodynamics for quantum systems described by mixed ensembles. The probability functions $P(\theta)$ and $Q(\vartheta)$ are found from probability amplitudes (see L1'). Accordingly, we can obtain the representation of the third law: The entropy $S_{\mathrm{m}}$ is equal to zero only when $P(\theta)$ and $Q(\vartheta)$ satisfy the conditions:

$$
\begin{gathered}
P(\theta ; x=i)=1 \text { for arbitrary } i, \\
Q(\vartheta ; y=j)=1 \text { for arbitrary } j .
\end{gathered}
$$

At the absolute zero temperature, one can expect a state of quantum system being in a single state for each ensemble $j$ such as

$$
\left|\Psi_{j}\right\rangle=b_{i j}|i\rangle \text { for arbitrary } i \text { and } j \text {. }
$$

The single state here means that only one outcome is obtained with a probability 1 by maximal tests.

\section{Summary and Concluding Remarks}

In this study, in addition to the parameters $\theta$ 's in a thermodynamic state space $\mathcal{M}_{\theta}$ to describe pure quantum ensembles discussed in Ref. [9], parameters $\vartheta$ 's 
in $\mathcal{M}_{\vartheta}$ are newly proposed in order to describe mixed quantum ensembles of quantum systems, where the map $\mathcal{M}_{\Psi} \mapsto \mathcal{M}_{\vartheta}$ is an injection. Therefore, in order to establish the representation of the zeroth law in terms of the elements $\mathcal{M}_{\vartheta}$, it is required to use the same maximal tests to obtain the corresponding elements from the respective elements of quantum state vectors $\Psi$ 's in the state-vector space $\mathcal{M}_{\Psi}$ for quantum systems. We showed that the first law is represented by a state quantity specified in terms of the parameters $\vartheta$ 's $\in \mathcal{M}_{\vartheta, L}$ along with the parameters $\theta$ 's $\in \mathcal{M}_{\theta, L}$ for the the systems in mixed ensembles (C1'), where $L$ denotes the work coordinate related to the work done on the respective system. We showed that the representation of the second law of thermodynamics was also obtained for a system in mixed quantum ensembles by using the parameters in $\mathcal{M}_{\vartheta}$. This representation obtained here could give some insight into the order relation in $\mathcal{M}_{\theta, L}$ and $\mathcal{M}_{\vartheta, L}$ for pure and mixed quantum systems. Therefore, the study of the order relation would afford one to investigate thermodynamic structure in a state space characterizing thermodynamics of quantum systems in terms of those parameters in $\mathcal{M}_{\theta, L}$ and $\mathcal{M}_{\vartheta, L}$. Finally, we note on the third law. In our representation, when the state is described by $\left|\Psi_{j}\right\rangle=b_{i j}|i\rangle$, where $b_{i j}$ is the probability amplitude in a mixed ensemble, viz. the state of system is characterized by a single state for each ensemble $j$, entropy of the system takes the value of zero. Suppose $|i\rangle$ be an energy eigenstate of the system. The state is then described by $\left|\Psi_{j}\right\rangle=b_{i j}|i\rangle$ at the absolute zero temperature. Thus we draw from third law that in our representation the entropy $S \rightarrow 0$ as temperature $T \rightarrow 0$, where the energy of the system $E_{i}$ corresponds to the lowest energy quantum state of the system. In summary, based on the mathematical foundation for quantum systems, we have obtained a representation of the basic laws (Zeroth, First, Second and Third Laws) in thermodynamics for quantum systems described by mixed quantum ensembles.

We hope that the present theory affords the mathematical foundation of the basic laws of quantum thermodynamics and the key to treat mixed quantum systems thermodynamically.

\section{Acknowledgements}

The authors appreciate unknown reviewers for their comments on the manuscript. One of the authors (H.T.) acknowledges the research grant from Hokkaido University of Education, which partly supported the present research.

\section{Conflicts of Interest}

The authors declare no conflicts of interest regarding the publication of this paper.

\section{References}

[1] Fermi, E. (1956) Thermodynamics, Dover, New York.

[2] Callen, H.B. (1985) Thermodynamics and an Introduction to Thermostatics. 2nd 
Edition, Wiley, New York.

[3] Lieb, E.H. and Yngvason, J. (1999) Physics Reports, 310, 1-96.

https://doi.org/10.1016/S0370-1573(98)00082-9

[4] von Neumann, J.L. (1932) Mathematische Grundlagen der Quantenmechanik, Springer, Berlin. In: Beyer, E.T., Ed., (1955) Mathematical Foundations of Quantum Mechanics, Princeton University Press, Princeton.

[5] Peres, A. (1993) Quantum Theory: Concepts and Methods. Kluwer-Academic, Boston.

[6] Wehrl, A. (1978) Reviews of Modern Physics, 50, 221. https://doi.org/10.1103/RevModPhys.50.221

[7] Partovi, M.H. (1989) Physics Letters A, 137, 440-444. https://doi.org/10.1016/0375-9601(89)90221-1

[8] Bender, C.M., Brody, D.C. and Meister, B.K. (2000) Journal of Physics A, 33, 4427-4436. https://doi.org/10.1088/0305-4470/33/24/302

[9] Suzuki, A. and Taira, H. (2018) Journal of Modern Physics, 9, 2420-2436. https://doi.org/10.4236/jmp.2018.914155

[10] Isham, C. J. (1995) Lectures on Quantum Theory: Mathematical and Structural Foundations, Imperial College Press, London. https://doi.org/10.1142/p001 